\title{
Psychothérapie effectuée par des psychologues: le manque de données comblé
}

Jusqu'à récemment, les informations disponibles sur l'offre, l'utilisation, les coûts et le financement des psychothérapies effectuées par des psychologues en Suisse n'étaient que parcellaires. Grâce à une étude mandatée par la Fédération Suisse des Psychologues (FSP), on dispose désormais de données sur le sujet. L'enquête atteste que les psychothérapeutes psychologues jouent un rôle important dans le traitement des troubles psychiques et peuvent contribuer à contrer la pénurie de psychiatres.

Yvik Adler ${ }^{a}$, Philipp Thüler ${ }^{b}$

a lic. phil., psychologue spécialiste en psychothérapie FSP et vice-présidente de la Fédération Suisse des Psychologues (FSP)

b Responsable adjoint du département Communication et marketing de la FSP

Liens d'intérêts:

L'étude a été principalement financée par la FSP.

Lire également à ce sujet le commentaire de la FMPP à la page 615 .

\footnotetext{
* Le rapport final complet peut être téléchargé sur www.psychologie.ch ou obtenu auprès de la FSP.
}

1 Giacometti-Bickel G, Landolt K, Bernath C, Seifritz E, Haug A Rössler W. Il manquera 1000 psychiatres en 10 ans. Bull Méd Suisses. 2013;94(8): 302-5.

Correspondance: Philipp Thüler Fédération Suisse des Psychologues (FSP) Choisystrasse 11

CH-3000 Berne 14 Tél. 0313888825 philipp.thueler[at]fsp. psychologie.ch
Les psychothérapeutes psychologues constituent un maillon indispensable du système de santé suisse. Compte tenu du manque de relève qui se dessine chez les psychiatres - un sujet déjà abordé dans ce magazine [1] - il semble clair que, sans intégrer de manière adéquate les psychothérapeutes psychologues, il risque d'être difficile de répondre à la demande.

La Loi sur les professions de la psychologie (LPsy), entrée en vigueur en avril 2013, fixe clairement les conditions de la pratique de la psychothérapie par les psychologues. La LPsy protège le titre de psychothérapeute psychologue. En toute logique, le modèle de la psychothérapie déléguée est donc voué à disparaître. L'Office fédéral de la santé public (OFSP) examine actuellement de nouveaux modèles d'admission de la psychothérapie effectuée par des psychologues dans l'assurance de base.

\section{Prescription au lieu de délégation?}

Le modèle existant de psychothérapie déléguée doit être remplacé par celui de la prescription, actuellement à l'étude. Selon ce modèle, le ou la psychologue psychothérapeute effectue le traitement prescrit par un médecin sous sa propre responsabilité et facture directement ses prestations via l'assurance de base.

\section{Des données de base solides}

Pour pouvoir s'appuyer sur des données de base solides et actuelles dans le cadre des discussions sur la définition d'un nouveau modèle, la FSP a mandaté le bureau d'études de politique du travail et de politique sociale BASS pour mener une enquête approfondie sur le sujet. Cette enquête a bénéficié du soutien d'autres associations professionnelles SBAP (Association Professionnelle Suisse de Psychologie Appliquée) et ASP (Association Suisse des Psychothérapeutes), leurs membres respectifs ainsi que SSPP (Société Suisse de Psychiatrie et Psychothérapie), FMPP (Fédération des médecins psychiatres-psychothérapeutes de Suisse, association faîtière) et ASMP
(Association suisse des médecins-cheffes et médecins-chefs en psychiatrie).

Le rapport final mis à disposition* contient des données sur le nombre de psychothérapeutes psychologues en activité en Suisse, sur l'utilisation de leurs prestations (qui et comment), sur le coût de celles-ci et sur leur mode de financement.

\section{Une enquête menée auprès de 9100 personnes}

L'enquête s'est déroulée en deux temps: quelque 9100 psychologues ont été interrogés au cours de la première phase. Le but de cette enquête exhaustive était d'en apprendre plus sur le nombre de psychologues actifs dans le domaine de la psychothérapie. Le cas échéant, il s'agissait également de déterminer le volume et le type d'activité correspondants (indépendante, déléguée, en institution ambulatoire ou en milieu hospitalier).

Au cours de la deuxième phase, 2200 personnes ont été sélectionnées parmi les 4000 psychologues actifs dans le domaine de la psychothérapie; elles ont été interrogées sur les prestations psychothérapeutiques qu'elles ont fournies durant l'année 2012. Les taux de retour élevés (67\% pour l'enquête exhaustive, 53\% pour l'enquête représentative) ont permis de produire des données extrêmement fiables. Grâce à des données secondaires obtenues gracieusement de SASIS SA, une filiale de santésuisse, les résultats ont en effet pu être comparés avec le coût de l'assurance de base. Cette comparaison confirme la fiabilité élevée des données recueillies. Les données de l'enquête ont en outre été complétées par des données relatives à l'assurance complémentaire d'un assureur-maladie important.

\section{0 psychothérapeutes psychologues}

Selon le rapport final de l'enquête environ 5700 psychothérapeutes psychologues ont fourni des prestations psychothérapeutiques en Suisse, pour environ 3100 équivalents plein temps. Leur taux d'occupa- 
Figure 1

Structure d'âge selon le type d'activité.

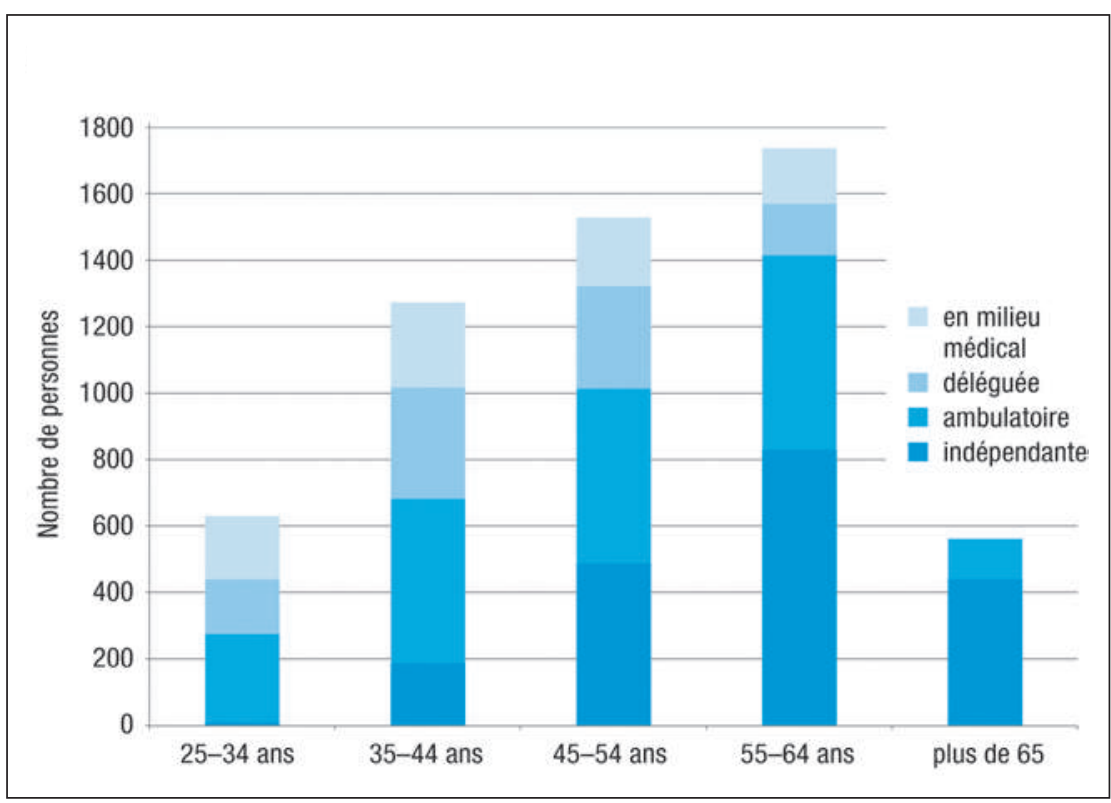

tion moyen a oscillé entre 70 et $90 \%$, un chiffre qui englobe également le temps consacré aux tâches administratives et de gestion, à la supervision et à la formation postgrade.

L'offre de psychothérapies effectuées par des psychologues s'est répartie comme suit: cabinets indépendants (34\%), PT déléguée (31\%) et institutions (36\%). Logiquement, les psychothérapeutes les plus jeunes ont travaillé plutôt de manière déléguée ou en institution qu'à titre indépendant. La majeure partie des psychothérapeutes indépendant-e-s s'est retrouvée dans la classe d'âge des 55 à 64 ans (fig. 1). La part de celles et de ceux qui maitrisent la langue de la région où ils ont pratiqué aussi bien que leur langue maternelle est extrêmement élevée (90 à 98\%).

La répartition par sexe a révélé que $75 \%$ des psychothérapeutes psychologues étaient des femmes, pour $25 \%$ d'hommes. Les méthodes de psychothérapie employées ont été très variées. Quelque $55 \%$ des psychothérapeutes psychologues ont déclaré avoir suivi une deuxième ou plusieurs formations postgrades en psychothérapie. L'étude apporte la preuve qu'avant l'entrée en vigueur de la LPsy, les psychothérapeutes psychologues présentaient déjà un niveau de qualification élevé, adapté au traitement psychothérapeutique de personnes atteintes de troubles psychiques.

\section{9 consultations en moyenne}

En 2012, les psychothérapeutes psychologues suisses ont dispensé quelque 3,1 millions de consultations à 260000 patient-e-s. Environ 60\% des patient-e-s ont suivi entre deux et vingt séances dans l'année, $21 \%$ des thérapies se poursuivant quant à elles sur 21 à 40 séances. Seule une toute petite minorité de patient-e-s, de l'ordre de $4 \%$, a eu recours à plus de 40 consultations dans l'année (tabl. 1, page suivante). La durée moyenne d'une thérapie a été de 29 séances sur une durée moyenne de 15 mois.

Le tableau 2 à la page suivante indique les motifs de traitement. Les patient-e-s ont été traités pour les catégories de troubles suivantes: troubles affectifs (25\%), troubles d'adaptation (25\%), troubles apparaissant durant l'enfance et l'adolescence (15\%), troubles de la personnalité (11\%), troubles liés à une dépendance (7\%) et troubles schizophréniques (3\%). Par ailleurs, $41 \%$ des patient-e-s ayant été traité-e-s par un psychothérapeute psychologue présentaient une comorbidité de troubles psychiques.

Pour résumer, la preuve a été apportée que les psychothérapeutes psychologues proposent une offre thérapeutique très riche couvrant l'ensemble de la gamme des troubles psychiques et traitent aussi des personnes atteintes de maladies psychiques graves.

\section{Accès à la psychothérapie}

Près de la moitié des psychothérapeutes psychologues ont signalé en 2012 l'existence de délais d'attente pour les nouveaux patients et/ou la nécessité de refuser des patient-e-s par manque de capacités. Quelque 16\% des patient-e-s ont renoncé à suivre une psychothérapie pour des raisons financières ou ont dû se tourner vers une thérapie remboursée par l'assurance de base. De plus, $22 \%$ des thérapies ont dû être prématurément interrompues pour les mêmes raisons. Ne sont pas recensées les personnes qui, compte tenu des conséquences financières, n'ont pas sollicité de psychothérapeute psychologue.

\section{Un taux d'autofinancement élevé}

En 2012, le coût des prestations fournies par des psychothérapeutes psychologues dans le secteur ambulatoire s'est élevé à 406 millions de francs. La majorité de ces coûts, soit un montant de 270 millions de francs, a été pris en charge par les assurances sociales, dont la plus grande partie (246 millions de francs) par l'assurance de base. L'assurance invalidité a, de son côté, payé 23 millions de francs. Un peu moins de 30\% du coût global des psychothérapies ambulatoires effectuées par des psychologues (117 millions de francs) ont été supportés par les patient-e-s eux-mêmes ou par les assurances complémentaires. Les extrapolations effectuées indiquent qu'une part très faible du coût des psychothérapies effectuées par des psychologues en 2012 (environ 18 millions de francs) a été prise en charge par les assurances complémentaires. Une proportion plus importante de ce coût (environ 100 millions de francs) a été supportée par les patient-e-s eux-mêmes.

\section{Conséquences d'un changement de système}

Quelles seraient les retombées d'un remplacement du modèle de la psychothérapie déléguée par celui de la prescription? A l'heure actuelle, nous ne connaissons pas encore précisément les modalités de fonctionnement du nouveau système. Il est donc difficile de faire des prévisions. Concernant la question des 
Tableau 1

Le nombre de patient-e-s ventilé selon le nombre de séances de thérapie et selon le type d'activité en 2012.

\begin{tabular}{|c|c|c|c|c|c|}
\hline & \multicolumn{2}{|l|}{ En cabinet } & \multicolumn{2}{|l|}{ En institution } & \multirow[t]{2}{*}{ Total } \\
\hline & PT indépendante & PT déléguée & PT ambulatoire & PT stationnaire & \\
\hline 1 séance/premier entretien & $12 \%$ & $9 \%$ & $21 \%$ & $27 \%$ & $16 \%$ \\
\hline 2-20 séances & $59 \%$ & $57 \%$ & $60 \%$ & $59 \%$ & $59 \%$ \\
\hline 21-30 séances & $17 \%$ & $18 \%$ & $11 \%$ & $9 \%$ & $14 \%$ \\
\hline 31-40 séances & $8 \%$ & $11 \%$ & $5 \%$ & $3 \%$ & $7 \%$ \\
\hline $41-50$ séances & $3 \%$ & $3 \%$ & $2 \%$ & $2 \%$ & $3 \%$ \\
\hline Plus de 51 séances & $2 \%$ & $1 \%$ & $1 \%$ & $1 \%$ & $1 \%$ \\
\hline Total & $100 \%$ & $100 \%$ & $100 \%$ & $100 \%$ & $100 \%$ \\
\hline $\begin{array}{l}\text { ( } \mathrm{n}=\text { nombre de personnes } \\
\text { qui ont répondu) }\end{array}$ & $n=441$ & $\mathrm{n}=373$ & $\mathrm{n}=172$ & $\mathrm{n}=118$ & $n=1104$ \\
\hline
\end{tabular}

Remarque: le tableau illustre uniquement les séances de thérapie qui ont eu lieu en 2012. II ne permet pas de calculer le nombre de séances par thérapie achevée.

(Source: Enquête structurelle, extrapolations BASS)

\section{Tableau 2}

La répartition des patient-e-s selon le tableau clinique en fonction de la classification CIM par rapport à la totalité des patient-e-s à valeur de maladie, en 2012, par type d'activité.

\begin{tabular}{|c|c|c|c|c|c|}
\hline & \multicolumn{2}{|l|}{ En cabinet } & \multicolumn{2}{|c|}{ En institution } & \multirow{2}{*}{$\begin{array}{l}\text { Total } \\
\text { e }\end{array}$} \\
\hline & $\begin{array}{l}\text { PT } \\
\text { indépendante }\end{array}$ & $\begin{array}{l}\text { PT } \\
\text { déléguée }\end{array}$ & $\begin{array}{l}\text { PT } \\
\text { ambulatoire }\end{array}$ & $\begin{array}{l}\text { PT } \\
\text { stationnaire }\end{array}$ & \\
\hline $\begin{array}{l}\text { Troubles mentaux organiques, y c. les troubles } \\
\text { symptomatiques (F00-F09) }\end{array}$ & $2 \%$ & $1 \%$ & $3 \%$ & $2 \%$ & $2 \%$ \\
\hline $\begin{array}{l}\text { Troubles mentaux et du comportement liés à l'utilisation } \\
\text { de substances psychoactives (F10-F19) }\end{array}$ & $2 \%$ & $4 \%$ & $11 \%$ & $13 \%$ & $7 \%$ \\
\hline $\begin{array}{l}\text { Schizophrénie, troubles schizotypiques et troubles délirants } \\
\text { (F20-F29) }\end{array}$ & $1 \%$ & $3 \%$ & $3 \%$ & $6 \%$ & $3 \%$ \\
\hline Troubles de l'humeur (affectifs) (F30-F39) & $23 \%$ & $31 \%$ & $19 \%$ & $33 \%$ & $26 \%$ \\
\hline $\begin{array}{l}\text { Troubles névrotiques, troubles liés à des facteurs de stress } \\
\text { et troubles somatoformes (F40-F48) }\end{array}$ & $31 \%$ & $30 \%$ & $24 \%$ & $22 \%$ & $27 \%$ \\
\hline $\begin{array}{l}\text { Syndromes comportementaux associés à des perturbations } \\
\text { physiologiques et à des facteurs physiques (F50-F59) }\end{array}$ & $4 \%$ & $3 \%$ & $3 \%$ & $4 \%$ & $3 \%$ \\
\hline Troubles de la personnalité et du comportement (F60-F69) & $10 \%$ & $14 \%$ & $11 \%$ & $8 \%$ & $11 \%$ \\
\hline Retard mental (F70-F79) & $2 \%$ & $1 \%$ & $2 \%$ & $1 \%$ & $2 \%$ \\
\hline Troubles du développement psychologique (F80-F89) & $3 \%$ & $2 \%$ & $4 \%$ & $1 \%$ & $3 \%$ \\
\hline $\begin{array}{l}\text { «Troubles du comportement et troubles émotionnels } \\
\text { apparaissant }\end{array}$ & $14 \%$ & $10 \%$ & $17 \%$ & $5 \%$ & $12 \%$ \\
\hline habituellement durant l'enfance et l'adolescence (F90-F98) & $14 \%$ & $10 \%$ & $17 \%$ & $5 \%$ & $12 \%$ \\
\hline Trouble mental, sans autre indication (F99) & $2 \%$ & $1 \%$ & $1 \%$ & $0 \%$ & $1 \%$ \\
\hline Autres troubles & $6 \%$ & $1 \%$ & $3 \%$ & $5 \%$ & $4 \%$ \\
\hline Total & $100 \%$ & $100 \%$ & $100 \%$ & $100 \%$ & $100 \%$ \\
\hline ( $n$ = nombre de personnes qui ont répondu) & $\mathrm{n}=434$ & $\mathrm{n}=372$ & $\mathrm{n}=173$ & $\mathrm{n}=116$ & $\mathrm{n}=1095$ \\
\hline
\end{tabular}

(Source: Enquête structurelle, extrapolations BASS)

coûts, on peut toutefois estimer sur la base des chiffres de l'étude qu'environ 131 millions de francs seraient transférés du domaine privé et public (financement personnel/assurances complémentaires et secteur public) à l'assurance de base. Ceci à condition que l'ensemble des coûts jusqu'ici supportés par le secteur privé soient transférés vers l'assurance de base et que le coût global des psychothérapies effectuées par des psychologues soit conforme à celui de 2012 .
Le nouveau système, destiné à remplacer la «solution transitoire» de la psychothérapie déléguée mise en place avant l'entrée en vigueur de la LPsy, doit améliorer les conditions de travail des psychothérapeutes psychologues. Seule la dissolution du système de délégation peut permettre aux psychothérapeutes psychologues de contribuer durablement à éviter une pénurie de l'offre et, ainsi, à favoriser la bonne santé mentale de la population suisse. 